\title{
PROMOVER LA INDAGACIÓN EN MATEMÁTICAS Y CIENCIAS: DESARROLLO PROFESIONAL DOCENTE EN PRIMARIA Y SECUNDARIA
}

\author{
(PROMOTING INQUIRY IN MATHEMATICS AND SCIENCE: PROFESSIONAL \\ DEVELOPMENT OF PRIMARY AND SECONDARY SCHOOL TEACHERS)
}

Francisco Javier García-García

Antonio Quesada-Armenteros

Marta Romero Ariza

Ana María Abril Gallego

Universidad de Jaén

DOI: $10.5944 / e d u c X X 1.23513$

Cómo referenciar este artículo/How to reference this article:

García-García, F. J.; Quesada-Armenteros, A.; Romero Ariza, M. y Abril Gallego, A. M. (2019). Promover la indagación en matemáticas y ciencias: desarrollo profesional docente en primaria y secundaria. Educación XX1, 22(2), 335-359, doi: 10.5944/educXX1.23513

García-García, F. J.; Quesada-Armenteros, A.; Romero Ariza, M. \& Abril Gallego, A. M. (2019). Promoting inquiry in Mathematics and Science: professional development of Primary and Secondary school teachers. Educación XX1, 22(2), 335-359, doi: 10.5944/educXX1.23513

\section{RESUMEN}

El presente estudio se ubica en la intersección de dos problemáticas: la renovación metodológica en la enseñanza de las ciencias y de las matemáticas hacia enfoques orientados a la indagación en el aula, y la formación realmente transformativa del profesorado. En el marco de un proyecto europeo, se diseñó un programa de desarrollo profesional sobre aprendizaje por investigación, implementado en 13 países europeos, y se diseñaron y validaron instrumentos para medir la evolución del profesorado que participaba en estos programas. En este artículo nos centramos en el caso español (18 programas de desarrollo profesional con docentes de primaria y secundaria). Se administró un cuestionario antes de la participación en el programa y tras la finalización del mismo, 
que abordaba diferentes dimensiones del aprendizaje por investigación (creencias, prácticas docentes, barreras y obstáculos percibidos). Además, el segundo cuestionario recogía la percepción del profesorado sobre el proceso de desarrollo profesional vivido y el impacto percibido en su práctica docente. Los resultados muestran ganancias del profesorado en todas las dimensiones analizadas acerca del aprendizaje por investigación, así como una mejora en la percepción de barreras y obstáculos que limitan el uso de esta metodología. Sin embargo, a pesar de la mejora observada, sus prácticas docentes aún no reflejan un uso extendido de esta metodología. Los resultados también muestran una valoración positiva de los procesos de desarrollo profesional vividos, y una percepción positiva del impacto en sus capacidades docentes, a pesar de una moderada transformación de sus prácticas. En conjunto, de los resultados extraemos conclusiones relevantes sobre dimensiones del aprendizaje por investigación que deben tratarse con mayor profundidad en programas de desarrollo profesional, así como sobre procesos de aprendizaje profesional en los que incidir para potenciar el carácter transformativo de los mismos.

\section{PALABRAS CLAVE}

Formación del profesorado; aprendizaje por investigación; enseñanza de las matemáticas; enseñanza de las ciencias; práctica pedagógica.

\section{ABSTRACT}

Our study is placed in the intersection of two problems: pedagogical renewal in the teaching of Science and Mathematics towards inquiryoriented perspectives, and the transformative professional development of teachers. Within a European project, a professional development program about inquiry-based learning was designed and implemented in 13 European countries. Tools to measure teachers' evolution within the program were also designed and validated. In this paper we focus on the Spanish case (18 professional development programmes implemented, with Primary and Secondary school teachers). They filled in a questionnaire before their participation in the programmes and when they finished. The questionnaire addressed different dimensions about inquiry-based learning (beliefs, teaching practices, perceived barriers and obstacles). Then, the second questionnaire addressed teachers' perceptions of the professional development process they experienced, and the impact teachers perceived on their teaching practices. Results show an increment in all of the dimensions analysed about inquiry-based learning, as well as an improvement in teachers' perception of barriers and obstacles that hinder 
the use of this pedagogy. However, despite the progress observed, teachers' practices do not reflect a wider use of this pedagogy as yet. Results also show that teachers positively value the professional development process experienced, with a positive perception of the impact of the programme on their teaching abilities, despite the moderate transformation they reported on their teaching practices. In summary, from the results, we extract relevant conclusions about inquiry-based learning dimensions that should be addressed deeply in professional development programmes, as well as about professional learning processes that should be enhanced to strengthen the transformative potential of such programmes.

\section{KEYWORDS}

Teacher education; inquiry based learning; mathematics education; science teaching; teaching practice.

\section{INTRODUCCIÓN}

En los últimos años, diversos informes, estudios internacionales e investigaciones han detectado un insuficiente interés de los jóvenes escolares hacia las ciencias y las matemáticas, en España (Couso et al., 2011), en Europa (Gago, 2004; Osborne \& Dillon, 2008) y en otros países (Potvin \& Hasni, 2014), alertando sobre sus consecuencias. El informe Encouraging STEM studies for the labour market (2015), elaborado a instancias del Parlamento Europeo, pone en evidencia la necesidad de trabajadores con sólidas habilidades en áreas científicas, tecnológicas, ingenieriles y matemáticas (áreas STEM en su acrónimo en inglés), destacando que «la tasa de desempleo de trabajadores con destrezas STEM ha sido muy baja, y menor que la tasa total de desempleo desde comienzo del 2000, incluso en países especialmente golpeados por la crisis como Grecia, Portugal y España» (Caprile, Palmén, Sanz \& Dente, 2015, p. 9, traducción propia).

Se trata de un problema complejo y multifacético, que debe ser abordado desde múltiples dimensiones: políticas educativas europeas y nacionales, diseño del currículo, formación inicial y continua del profesorado, cuestionamiento y renovación de las metodologías docentes, percepción social de la relevancia de la ciencia y de las matemáticas, relaciones entre la industria y los sistemas de enseñanza, diseño de materiales educativos que apoyen nuevas concepciones de las ciencias y de su aprendizaje, etc. 
En este artículo nos centraremos en dos de estas dimensiones: la renovación de metodologías docentes y la formación del profesorado, que abordaremos de forma conectada.

\section{Renovación metodológica: el aprendizaje por investigación}

Desde hace tiempo se postula la necesidad de modificar la enseñanza de las ciencias y las matemáticas orientando más las prácticas docentes hacia el fomento de procesos de investigación en el aula y de resolución de problemas (Anderson, 2002; Couso et al., 2011). Nos referiremos a este tipo de metodologías como de aprendizaje por investigación (inquiry-based learning, IBL en inglés).

Artigue y Blomhøj (2013) señalan que no hay una forma única de definir lo que se entiende por IBL. En ciencias, la definición más extendida es la de los estándares del National Research Council (2012):

una actividad polifacética que incluye la observación, la formulación de preguntas, la búsqueda de información en libros y otras fuentes para conocer lo que ya se sabe sobre un tema, el diseño y planificación de investigaciones, la revisión de ideas atendiendo a la evidencia experimental disponible, el manejo de herramientas asociadas a la adquisición, análisis e interpretación de datos, la formulación de respuestas, explicaciones y predicciones y la comunicación de resultados. La indagación requiere la identificación de asunciones, la aplicación del pensamiento lógico y crítico y la consideración de explicaciones alternativas. (NRC, 2012, p. 20, traducción propia)

En matemáticas, tras una revisión de marcos teóricos relevantes en la investigación en Didáctica de las Matemáticas, Artigue y Blomhøj (2013) proponen la siguiente adaptación:

Las prácticas de [aprendizaje basado en la investigación] en matemáticas incluyen diferentes tipos de actividades combinadas en procesos de indagación: elaboración de cuestiones; resolución de problemas; modelización y matematización: búsqueda de recursos e ideas; exploración; análisis de documentos y de datos; experimentación; formulación de conjeturas; ensayar, explicar, razonar, argumentar y probar; definir y estructurar; conectar, representar y comunicar. (Artigue \& Blomhøj, 2013, p. 808, traducción propia)

Ambas definiciones ponen el acento en los procesos, bien de las matemáticas, bien de las ciencias, y serán tomadas como nuestras definiciones de referencia. 
Según concluye Romero-Ariza (2017), un uso más generalizado del IBL podría mejorar la alfabetización científica y matemática de nuestros jóvenes. Además, tendría un impacto positivo en la implicación del alumnado y en su interés por las ciencias (McConney, Oliver, Woods-McConney, Schibeci \& Maor, 2014), redundando en una disposición positiva hacia estudios y vocaciones profesionales relacionas con la ciencia (Couso et al., 2011; Rocard et al., 2007).

La implementación del IBL en el aula supone un cambio profundo en los roles de profesorado y alumnado. Anderson (2002) señala que el profesor pasa de ser un «dispensador de conocimiento» a un "facilitador», y los estudiantes pasan de ser «receptores pasivos» a «gestores de su aprendizaje».

La adopción del IBL por parte del profesorado no es sencilla. Un estudio exploratorio realizado por Engeln, Euler y Maa $\beta$ (2013) a nivel europeo, con profesorado de matemáticas y ciencias de primaria y secundaria, detectó que el IBL no tenía aún un peso importante en el conjunto de prácticas diarias de aula. Y a pesar de que factores contextuales, como los currículos vigentes y las orientaciones nacionales sobre la enseñanza de las ciencias y las matemáticas, apoyan el uso del IBL en clase (Dorier \& García, 2013), el profesorado del estudio aún percibía obstáculos importantes.

Aunque son muchos los factores que determinan un uso más o menos extendido de una metodología, las creencias del profesorado (Glackin, 2016), y la formación a la que tenga acceso (Rocard et al., 2007), son de especial importancia.

\section{Crecimiento y desarrollo profesional del profesorado}

La Comisión Europea, en la introducción del documento Principios Comunes Europeos para las Competencias y Cualificaciones del Profesorado (European Comission, 2009), señala que el profesorado desempeña un papel decisivo para apoyar las experiencias de aprendizaje de los jóvenes, y que es un actor clave en la evolución de los sistemas educativos y en la implementación de reformas. Resulta pues crucial entender cómo evolucionan y cambian creencias y prácticas docentes del profesorado, pero también cómo estructurar propuestas de desarrollo profesional que apoyen este cambio de forma efectiva.

Históricamente, el cambio en las creencias y las prácticas docentes del profesorado se ha vinculado a cursos de formación, que pretendían cualificar al profesorado en el aprendizaje de ciertas destrezas profesionales aún no desarrolladas. Hoy en día se interpretan más procesos de aprendizaje 
permanente y de desarrollo de prácticas profesionales, lo que ha tenido un impacto en cómo se organiza la formación del profesorado, evolucionando hacia propuestas en las que el profesorado es un aprendiz activo (Thurlings \& den Brok, 2017), que va dando forma a su crecimiento profesional a través de prácticas reflexivas y de su participación en programas de desarrollo profesional (Clarke \& Hollingsworth, 2002).

Todo programa de desarrollo profesional pretende, en última instancia, desarrollar las capacidades del profesorado para promover cierto tipo de aprendizaje en el alumnado, a través de una ampliación de conocimientos, destrezas, actitudes y creencias. Guskey (2002) considera crucial centrarse en las experiencias de aplicación de nuevas metodologías en el aula, como precursoras de un cambio en las creencias y actitudes del profesorado (Figura 1), entendiendo que experiencias de implementación exitosa conducirán a cambios en creencias y actitudes.

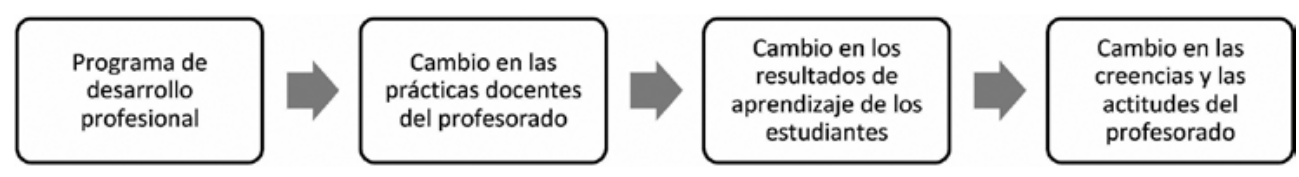

Figura 1. Modelo de cambio en el profesor

Fuente: Guskey, 2002, p. 383, traducción propia

Otros autores proponen un modelo no lineal de cambio del profesorado, que sugiere que este ocurre a través de los procesos de reflexión y de aplicación, dentro de cuatro dominios diferentes que conforman el mundo del profesor (Figura 2).

Igualmente, Jones y Eick (2007) consideran que hay una retroacción y una interacción mutua entre lo que el profesorado piensa y lo que experimenta. Loucks-Horsley, Love, Stiles, Mundry y Hewson (2003) describen esta interacción como una espiral en la que creencias y acciones evolucionan de forma conjunta, en un sistema complejo de intercambio de factores que conforman el sistema de creencias y el conocimiento práctico del profesor.

También las creencias de autoeficacia del profesorado son cruciales en la puesta en práctica de reformas educativas. Un nivel bajo de confianza en su conocimiento o en sus destrezas profesionales puede hacer que el profesorado sea más reacio a nuevas metodologías, ya que estas implican un reto y un menor nivel de control (Donnel \& Gettinger, 2015; Lakshmanan, Heath, Perlmutter \& Elder, 2011). 


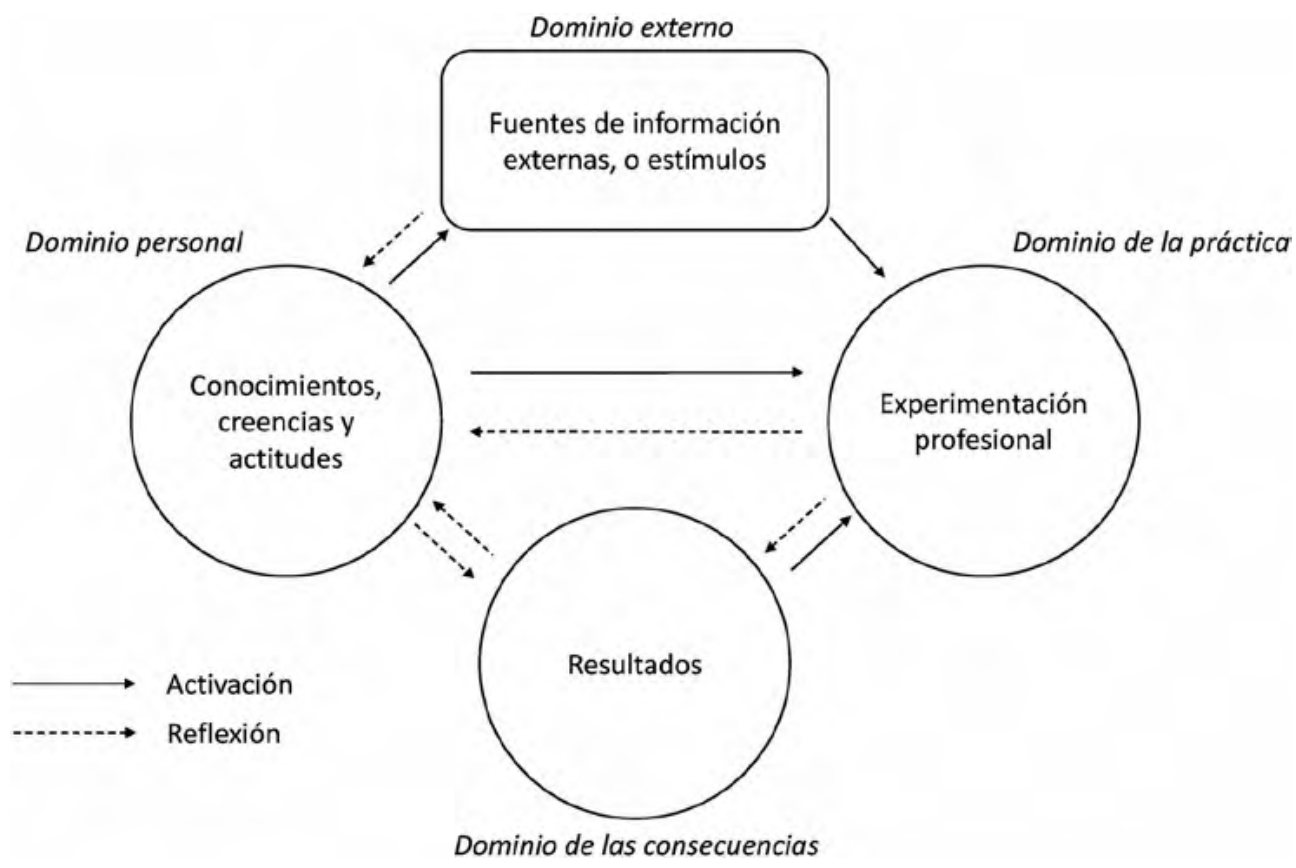

Figura 2. Modelo de crecimiento profesional

Fuente: Clarke \& Hollingsworth, 2002, p. 951, traducción propia

Según Bandura (ver Lakshmanan et al., 2011), las experiencias de dominio son las más poderosas para influir en las creencias de autoeficacia. Tener éxito en la ejecución de una determinada tarea las hace crecer de forma significativa. Esa correlación explica por qué las creencias están tan fuertemente conectadas con las experiencias y por qué la influencia entre creencias y prácticas docentes es bidireccional. También las experiencias de observación son importantes: observar a otros implementando IBL de forma satisfactoria puede provocar un incremento de actitudes positivas hacia esta metodología.

El diseño de propuestas de desarrollo profesional que impulsen un crecimiento real en el profesorado debe ser coherente con los principios de cambio descritos por la literatura, intentando provocar una interacción fecunda entre experiencias y creencias del profesorado. Según Tirosh y Graeber (2003), un programa de desarrollo profesional efectivo debe tener ciertas características clave, tanto en sus contenidos como en los procesos de formación utilizados (Tabla 1). 
Tabla 1

Desarrollo profesional del profesorado: principios de efectividad (adaptado de Tirosh \& Graeber, 2003)

\begin{tabular}{l|l}
\hline \multicolumn{1}{c|}{ Contenidos } & \multicolumn{1}{c}{ Procesos } \\
\hline $\begin{array}{l}\text { Tener como objetivo fundamental } \\
\text { el aprendizaje de los estudiantes y } \\
\text { cómo este se produce. }\end{array}$ & $\begin{array}{l}\text { Reflejar los principios metodológi- } \\
\text { cos que se espera que los profesores } \\
\text { pongan en funcionamiento con sus } \\
\text { alumnos. }\end{array}$ \\
\hline $\begin{array}{l}\text { Estar fundamentado en el cono- } \\
\text { cimiento matemático [respectiva- } \\
\text { mente, de ciencias] que los profeso- } \\
\text { res tienen que enseñar. }\end{array}$ & $\begin{array}{l}\text { Fomentar y desarrollar la colabora- } \\
\text { ción entre el profesorado. }\end{array}$ \\
\hline $\begin{array}{l}\text { Estar conectado con las prácticas } \\
\text { docentes del profesorado, sirviendo } \\
\text { de apoyo a las mismas. }\end{array}$ & $\begin{array}{l}\text { Tener en cuenta y usar el conoci- } \\
\text { miento y la experiencia del profeso- } \\
\text { rado. }\end{array}$ \\
\hline $\begin{array}{l}\text { Estar de acuerdo con el contexto en } \\
\text { el que los profesores trabajan. }\end{array}$ & $\begin{array}{l}\text { Crear algún tipo de desequilibrio o } \\
\text { insatisfacción con ciertas prácticas } \\
\text { docentes y/o resultados de aprendi- } \\
\text { zaje infructuosos, provocando una } \\
\text { necesidad de cambio. }\end{array}$ \\
\hline & $\begin{array}{l}\text { Partir de una cultura profesional } \\
\text { que apoya la formación del profe- } \\
\text { sorado, ofreciendo tiempo y espacio } \\
\text { para facilitar el cambio. }\end{array}$ \\
\cline { 2 - 2 } & $\begin{array}{l}\text { Evitar la intervención puntual } \\
\text { y anecdótica, extendiéndose en el } \\
\text { tiempo. }\end{array}$ \\
\hline
\end{tabular}

Ante la diversidad de modelos de desarrollo profesional, Kennedy (2014) propone una clasificación atendiendo a su potencial para modificar las prácticas del profesorado y a la autonomía de la que dote al profesorado, distinguiendo entre modelos:

- Transmisivos: normalmente organizados e impartidos lejos del aula, administrados por un "experto», con poca autonomía para el profesorado y débilmente vinculados con el contexto en el que este actúa. Concebidos principalmente para completar un «déficit» en el conocimiento del profesorado y con un flujo unidireccional de información. Suelen tener un impacto muy limitado en sus prácticas futuras. 
- Transicionales: fomentan la colaboración y la reflexión, usando tanto actividades prácticas como la propia práctica docente en el aula. Consideran el contexto en el que trabaja el profesorado, fomentando su autonomía y conectando con prácticas reales en el aula. No se centran en la transmisión de conocimientos, aunque pueden incluir episodios transmisivos. Se espera que tengan cierto impacto en las prácticas docentes del profesorado.

- Transformativos: fuertemente basados en la colaboración entre el profesorado y en la reflexión sobre su práctica docente, dotándolo de una gran autonomía (tendiendo hacia la auto-regulación). Vinculados a un contexto determinado, que condiciona y dirige el proceso, e insertados en prácticas reales de aula, incluyendo episodios de investigación. Provocan un verdadero cambio en las prácticas docentes del profesorado.

En cualquier caso, esta caracterización de posibles modelos de desarrollo profesional debe ser entendida como apriorística e ideal. Nos ofrece un marco de referencia para diseñar y planificar propuestas de desarrollo profesional más o menos transformativas, pero cuyo impacto real deberá ser analizado y medido en cada caso.

\section{METODOLOGÍA}

El presente estudio se centra en un programa de desarrollo profesional del profesorado de matemáticas y ciencias, desarrollado e implementado dentro de un proyecto de investigación europeo. Se articula en torno a las siguientes preguntas de investigación:

- ¿Qué presencia tiene el IBL en las creencias, actitudes y en las prácticas docentes del profesorado?

- ¿Cómo percibe el profesorado ciertas dificultades para el uso del IBL?

- ¿Hasta qué punto la forma en la que el programa se implementó fomentó la colaboración, reflexión y la participación activa del profesorado?

- ¿Qué cambios ha experimentado el profesorado en sus creencias, actitudes y prácticas tras su participación en un programa de desarrollo profesional?

\section{Diseño de un programa de desarrollo profesional}

El proyecto Mascil (Mathematics and Science for Life!, 7. ${ }^{\circ}$ Programa Marco) tuvo, entre sus objetivos, promover un uso más extendido del IBL en matemáticas y ciencias en centros de primaria y secundaria en Europa, 
diseñando y desarrollando una variedad de acciones dirigidas a diferentes destinatarios. Aquí nos centraremos en el diseño e implementación de un programa de desarrollo profesional.

Tomando como referencia los resultados de investigación descritos en el apartado anterior, se adoptó el modelo de comunidades profesionales de aprendizaje (Hord \& Hirsh, 2008). Para apoyar el desarrollo de estas comunidades, se diseñaron un conjunto de instrumentos (toolkit) estructurado en tres dominios: (1) cómo trabajar como una comunidad; (2) el mundo del trabajo (conexiones entre este, las matemáticas y las ciencias, y su enseñanza); y (3) IBL y metodologías. Se siguió una estructura jerárquica, de manera que cada dominio se dividió en un conjunto de temas, estos en un conjunto de cuestiones profesionales, y estas en diferentes herramientas (Figura 3). El objetivo era que, con la ayuda de estas herramientas, la comunidad de aprendizaje pudiese elaborar posibles respuestas a las cuestiones profesionales propuestas, que a su vez contribuyesen a desarrollar su conocimiento y competencias profesionales en relación con los temas y dominios identificados.

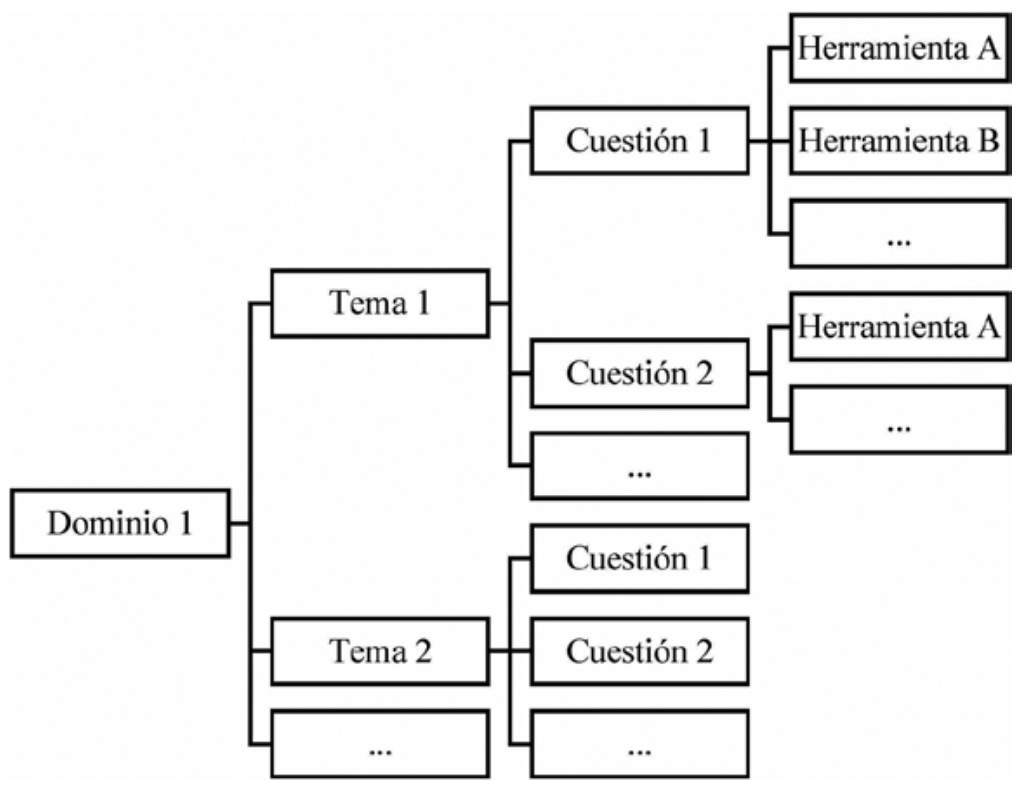

Figura 3. Estructura de los materiales de Mascil para la formación del profesorado

Por ejemplo, en el dominio "IBL y metodologías» se identificaron tres temas: (1) una clase orientada a la investigación, (2) el IBL en matemáticas y (3) el IBL en ciencias. La Tabla 2 describe las cuestiones profesionales y las herramientas diseñadas para explorar el primer tema. 
Tabla 2

Materiales de Mascil: desarrollo del tema «una clase orientada a la investigación $(I B L) »$

\begin{tabular}{c|l}
\hline Cuestiones profesionales & \multicolumn{1}{c}{ Herramientas } \\
\hline $\begin{array}{c}\text { ¿Qué ocurre en una } \\
\text { clase IBL? }\end{array}$ & $\begin{array}{l}\text { Características de una clase IBL } \\
\text { Observación de una clase IBL }\end{array}$ \\
\hline ¿Funciona el IBL? & $\begin{array}{l}\text { Exploración de los beneficios del IBL } \\
\text { Exploración de evidencias }\end{array}$ \\
\hline $\begin{array}{c}\text { ¿Cuáles son los retos } \\
\text { a afrontar al usar IBL? }\end{array}$ & $\begin{array}{l}\text { Identificación de barreras y dilemas } \\
\text { Evaluación del aprendizaje por investigación }\end{array}$ \\
\hline \multirow{2}{*}{ unámo se apoya } & $\begin{array}{l}\text { El uso de cuestiones en clase (discusión) } \\
\text { El uso de cuestiones en clase (juego de rol) } \\
\text { Planificación del uso efectivo de cuestiones } \\
\text { El trabajo colaborativo de los estudiantes }\end{array}$ \\
\hline
\end{tabular}

Los materiales están dirigidos a un facilitador de la comunidad de aprendizaje profesional, entendiendo que este papel lo podría desempeñar un profesional externo (formador), o bien algún miembro de la propia comunidad de profesores en formación. Las herramientas no se diseñaron como piezas de información a transmitir, aunque algunas pueden incluir breves episodios transmisivos (en el sentido de Kennedy, 2014), sino para fomentar la discusión, la reflexión y la participación activa del profesorado, bien dentro de la comunidad, bien en su clase. Por ejemplo: la herramienta "Características de una clase IBL» tiene por objetivo hacer emerger las creencias de la comunidad sobre las características básicas de una clase IBL, a través de una discusión entre el profesorado articulada en torno a dos cuestiones guía: ¿qué hacen los estudiantes en una clase IBL?, ¿y el docente?

Con estos materiales, se llevaron a cabo procesos de desarrollo profesional en 13 países europeos. Las implementaciones concretas variaron en la selección y el uso de los materiales, ya que la propuesta de Mascil es abierta en cuanto a los temas y cuestiones a explorar, y las herramientas a usar. A continuación, esbozaremos cómo se llevó a cabo en España.

\section{La intervención de Mascil en España}

En España se implementaron 18 programas de desarrollo profesional, entre junio de 2014 y marzo de 2016, con profesorado tanto de matemáticas como de ciencias, en los que en total participaron más de 300 docentes de primaria y secundaria. Aunque existen múltiples diferencias entre ambos colectivos, 
los programas se diseñaron con el objetivo de integrar profesorado de ambas etapas y disciplinas, poniendo el acento más en los procesos de investigación que en los contenidos disciplinares, y asumiendo que las metodologías que apoyan el aprendizaje por investigación tienen rasgos comunes.

Así, respecto a los contenidos, los programas se centraron en el dominio "IBL y metodologías», abordando aspectos relacionados con las características del IBL (tipos de tareas, roles del profesorado y del alumnado, retos) y con metodologías que apoyan el IBL (uso de cuestiones y trabajo colaborativo). También se usaron materiales relacionados con los temas «IBL en matemáticas» e "IBL en ciencias», principalmente para la exploración de actividades IBL y para el diseño de otras nuevas.

Un aspecto crucial de las 18 intervenciones fue la combinación de sesiones de trabajo en grupo con momentos de implementación en el aula. Todas incluyeron la necesidad de escoger o diseñar una o varias tareas IBL, y de usarlas en clases reales, aunque la forma concreta de hacerlo dependió de la estructura final de cada programa. En tres de ellos se impartieron sesiones presenciales (entre 2 y 5 , en torno a 3 h de duración por sesión) intercaladas con periodos de experimentación en el aula (en total, estos oscilaban entre 1 y 3 meses). En once casos se hizo una sesión presencial intensiva ( $8 \mathrm{~h}$ ) seguida de un periodo autónomo de implementación en el aula. En cuatro, uno o varios días presenciales (hasta 5 días) precedían una fase online (de aproximadamente 2 meses). Durante estos periodos de diseño e implementación el profesorado puede apropiarse de las metodologías que promueven la investigación en el aula, adaptándolas a las características de su alumnado (de primaria o secundaria), y a la disciplina que imparta (en secundaria) o en la que se centre (en primaria).

Según el diseño de los materiales Mascil, los procesos de desarrollo profesional se orientaron más hacia comunidades de aprendizaje guiadas por un experto que a cursos expositivos tradicionales. Se usaron dinámicas de trabajo en grupo y colaborativo, buscando la construcción compartida de conocimiento profesional y fomentando la reflexión.

\section{Muestra e instrumento}

Se ha utilizado una metodología cuasi-experimental con grupo pre-test post-test con el objeto de indagar sobre el impacto del curso partiendo de una base de comparación apropiada. La muestra del estudio es intencional, formada por el profesorado implicado en los programas de formación que completó los cuestionarios antes de comenzar (226) y al finalizar los mismos (117) (Tabla 3). En 52 casos fue posible establecer emparejamientos entre el pre-test y el post-test. La mayoría del profesorado proviene de centros públicos y concertados de Andalucía. 
Tabla 3

Características de la muestra

\begin{tabular}{l|cc|cccc|c|cc}
\hline & \multicolumn{2}{|c|}{ Sexo } & \multicolumn{2}{c|}{ Experiencia profesional (años) } & \multicolumn{2}{c|}{$\begin{array}{c}\text { Profesorado } \\
\text { Primaria }\end{array}$} & \multicolumn{2}{c}{$\begin{array}{c}\text { Profesorado } \\
\text { Secundaria }\end{array}$} \\
\cline { 2 - 10 } & $\mathbf{H}$ & $\mathbf{M}$ & $\mathbf{2 5}$ & $\mathbf{5 < 1 0}$ & $\mathbf{1 1 < 2 0}$ & $\mathbf{> 2 0}$ & - & Matem. & Ciencias \\
\hline $\mathbf{N}_{\text {pre-test }}=\mathbf{2 2 6}$ & $48,8 \%$ & $51,2 \%$ & $15,1 \%$ & $24,9 \%$ & $25,3 \%$ & $34,6 \%$ & $51,9 \%$ & $72,5 \%$ & $27,5 \%$ \\
$\mathbf{N}_{\text {post-test }}=\mathbf{1 1 7}$ & $36,3 \%$ & $63,7 \%$ & $11,2 \%$ & $24,0 \%$ & $29,6 \%$ & $35,2 \%$ & $79,8 \%$ & $68,2 \%$ & $31.8 \%$ \\
$\mathbf{N}_{\text {pareada }}=\mathbf{5 2}$ & $36,5 \%$ & $63,5 \%$ & $11,5 \%$ & $25,0 \%$ & $30,8 \%$ & $32,7 \%$ & $92,5 \%$ & $50,0 \%$ & $50,0 \%$ \\
\hline
\end{tabular}

El análisis de datos se ha realizado con el paquete estadístico SPSS v24

El instrumento utilizado es una versión traducida al castellano del cuestionario desarrollado en el proyecto Mascil. El cuestionario original, en inglés, ha sufrido diversos ciclos de refinado y en sus diferentes versiones ha sido pilotado con una elevada muestra internacional de profesorado $(\mathrm{N}=1315)$. El cuestionario utiliza una escala tipo Likert. El profesorado ha de indicar, según la dimensión analizada, su grado de acuerdo/descuerdo $(1$ = totalmente en desacuerdo; 4 = totalmente de acuerdo $)$ o frecuencia ( 1 = nunca; 4 = siempre). Comienza con una sección autobiográfica: datos personales y experiencia profesional del profesorado. El resto de secciones incluyen ítems relacionados con las creencias del profesorado sobre diversas dimensiones del IBL, su práctica docente, y sobre barreras que perciben para el uso del IBL. Además, el cuestionario post-test incluye ítems para medir la valoración y percepción de los participantes en relación con determinados aspectos del programa de desarrollo profesional que vivieron. Para una descripción más detallada, en su versión original, incluyendo información sobre el diseño, elección y redacción de los ítems, escalas y validación del mismo, véase Engeln (2017).

Los diferentes ítems se han agrupado en escalas con la intención de medir diferentes dimensiones. En primer lugar, se han considerado dos dimensiones, una relacionada con rutinas de implementación del IBL en el aula (RUT) y otra con actitudes favorables hacia el IBL (ORI). Ambas escalas son fundamentales ya que, según se ha indicado en el marco teórico, la relación entre creencias y prácticas docentes es crucial en todo programa de desarrollo profesional (Clarke \& Hollingsworth, 2002; Jones \& Eick, 2007; Loucks-Horsley et al., 2003).

El uso en clase del IBL debería implicar una mayor frecuencia de empleo de ciertas estrategias metodológicas (Anderson, 2002); por un lado, estrategias más centradas en los alumnos, que ofrecen un mayor grado de autonomía y de responsabilidad (por ejemplo, «explicar sus ideas», «discutir sobre temas de interés» o «elegir las cuestiones sobre las que 
desean investigar»). Por otro lado, un mayor uso de actividades prácticas y la realización de trabajo experimental. Por ello, en el cuestionario se incluye un conjunto de ítems orientados a medir con qué frecuencia aparecen estos aspectos en las metodologías usadas por el profesorado:

- CE-EST: frecuencia con la que usan prácticas docentes centradas en los estudiantes.

- PRA: frecuencia con la que usan actividades prácticas.

- INV: frecuencia con la que llevan a cabo investigaciones en el aula.

En la comparación pre-post deseamos determinar el impacto del programa Mascil en la frecuencia de uso de estas metodologías en el profesorado que ha participado en los cursos.

Otros aspectos importantes son los relacionados con las oportunidades, barreras y dificultades que percibe el profesorado para el uso del IBL. Se han agrupado en tres escalas:

- LEG: oportunidades que ofrece el contexto legislativo (presencia y apoyo del currículo al uso del IBL).

- GEST: barreras y dificultades percibidas relacionadas con la gestión del aula. Por cómo fueron formulados los ítems en esta escala (por ejemplo, «el IBL es difícil para mis alumnos», o «es difícil gestionar una clase IBL»), una tendencia hacia el desacuerdo debe ser interpretada como positiva. Para favorecer la interpretación de los datos, para esta dimensión indicaremos los valores de la escala original (GEST) y también de la escala invertida (GEST $\left.{ }^{\text {inv }}\right)$.

- REC: barreras y dificultades percibidas relacionadas con los recursos y materiales de aula disponibles.

La comparación pre-post nos permitirá determinar la influencia de los programas en la percepción de estas oportunidades y dificultades por parte del profesorado.

Finalmente, un conjunto de ítems incluidos solo en el post-test pretenden medir la percepción del profesorado acerca de las metodologías usadas en el programa de desarrollo profesional en el que participaron (escala MET-DP), así como la significatividad del mismo (escala REL-DP) en términos de aprendizajes relevantes y útiles para su labor docente.

Los análisis de fiabilidad del instrumento (Tabla 4) para las escalas definidas utilizando el alfa de Cronbach arrojaron resultados en el rango 0,926-0,564. Según Huh, Delorme y Reid (2006), estos valores pueden ser considerados aceptables para abordar el estudio por escalas, y el alcance del 
mismo planteado, asumiendo que los ítems miden un mismo constructo y entre ellos existe cierta correlación.

Tabla 4

Descripción de los elementos del cuestionario y fiabilidad

\begin{tabular}{lcllc}
\hline Nombre & $\begin{array}{c}\text { N.o de } \\
\text { items }\end{array}$ & \multicolumn{1}{c}{ Descripción } & \multicolumn{1}{c}{ Tipo de escala } & $\begin{array}{c}\text { Alfa } \\
\text { Cronbach }\end{array}$ \\
\hline RUT & 3 & Rutina e IBL & Desacuerdo/Acuerdo & 0,823 \\
ORI & 3 & Orientación hacia el IBL & Desacuerdo/Acuerdo & 0,682 \\
CE-EST & 5 & $\begin{array}{l}\text { Oportunidades para el } \\
\text { alumnado }\end{array}$ & Frecuencia & 0,770 \\
PRA & 3 & Actividades prácticas & Frecuencia & 0,586 \\
INV & 3 & Investigación & Frecuencia & 0,729 \\
LEG & 5 & Contexto legislativo e IBL & Desacuerdo/Acuerdo & 0,705 \\
GEST & 3 & Gestión de un aula IBL & Desacuerdo/Acuerdo & 0,636 \\
REC & 2 & Materiales e IBL & Desacuerdo/Acuerdo & 0,564 \\
MET-DP & 9 & Metodologías en el CDP & Desacuerdo/Acuerdo & 0,868 \\
REL-DP & 14 & Satisfacción del CDP & Desacuerdo/Acuerdo & 0,926 \\
\hline
\end{tabular}

\section{RESULTADOS Y DISCUSIÓN}

La Tabla 5 recoge los principales resultados del análisis pre-test posttest realizado por dimensión, y no por ítems. Los datos se han normalizado teniendo en cuenta el número de ítems para cada una de las escalas definidas en la Tabla 4. La prueba de comparación de medias (t-Student) arrojó resultados de diferencias significativas $(* p<0,05, * * p<0,001)$ en algunas de las dimensiones analizadas.

Por rigor estadístico, el análisis y la discusión la llevaremos a cabo con los resultados de la muestra pareada, si bien podría ser plausible su extrapolación a toda la muestra, teniendo en cuenta la similitud obtenida en los resultados (Tabla 5). En líneas generales se observa un aumento en los valores de todas las dimensiones analizadas, lo que nos permite interpretar de forma positiva la evolución de creencias y prácticas del profesorado sobre diferentes aspectos del IBL.

Los resultados más relevantes apuntan a diferencias entre el grupo pretest y el post-test con determinado grado de significatividad en las dimensiones 
relacionadas con las escalas rutina de implementación de IBL (RUT), dificultades para la gestión del aula IBL (GEST), oportunidades de investigación que se ofrece al alumnado (INV) y disponibilidad de materiales IBL (REC).

Tabla 5

Valores pre-test y post-test para la muestra del estudio (pareada y global)

\begin{tabular}{|c|c|c|}
\hline \multirow{3}{*}{ Dimensión } & \multicolumn{2}{|c|}{ Muestra global } \\
\hline & \multicolumn{2}{|c|}{ Media (desviación estándar) } \\
\hline & Pre $(N=226)$ & Post $(N=117)$ \\
\hline RUT $^{* * *}$ & $2,00(0,53)$ & $2,28(0,49)$ \\
\hline ORI* & $3,19(0,42)$ & $3,29(0,41)$ \\
\hline CE-EST & $2,28(0,56)$ & $2,39(0,52)$ \\
\hline PRA & $2,12(0,54)$ & $2,18(0,72)$ \\
\hline $\mathrm{INV}^{* *}$ & $1,57(0,49)$ & $1,78(0,49)$ \\
\hline LEG & $2,18(0,40)$ & $2,20(0,37)$ \\
\hline GEST** & $2,47(0,55)$ & $2,27(0,43)$ \\
\hline GEST $^{\text {inv*** }}$ & $2,53(0,43)$ & $2,73(0,42)$ \\
\hline REC* & $1,84(0,47)$ & $2,05(0,50)$ \\
\hline MET-DP & - & $2,99(0,40)$ \\
\hline REL-DP & - & $3,02(0,40)$ \\
\hline
\end{tabular}

\begin{tabular}{lcc}
\hline \multirow{2}{*}{ Dimensión } & \multicolumn{2}{c}{ Muestra pareada } \\
\cline { 2 - 3 } & \multicolumn{2}{c}{ Media (desviación estándar) } \\
\cline { 2 - 3 } & Pre $(\mathbf{N}=\mathbf{5 2})$ & Post $(\mathbf{N}=\mathbf{5 2})$ \\
\hline RUT ** $^{* *}$ & $1,96(0,46)$ & $2,30(0,45)$ \\
ORI & $3,22(0,37)$ & $3,30(0,43)$ \\
CE-EST & $2,23(0,53)$ & $2,47(0,49)$ \\
PRA & $2,18(0,55)$ & $2,22(0,45)$ \\
INV** & $1,50(0,43)$ & $1,84(0,46)$ \\
LEG & $2,11(0,45)$ & $2,21(0,37)$ \\
GEST* & $2,48(0,42)$ & $2,25(0,45)$ \\
GEST ${ }^{\text {inv** }}$ & $2,52(0,43)$ & $2,75(0,48)$ \\
REC* & $1,83(0,41)$ & $2,07(0,54)$ \\
MET-DP & - & $2,95(0,39)$ \\
REL-DP & - & $3,01(0,37)$ \\
\hline
\end{tabular}

Fuente: elaboración propia

El análisis de los resultados de la escala ORI (actitudes) pone de manifiesto un elevado valor de partida en el profesorado implicado en el curso. Este valor de partida conduce a pensar que en el post-test no se debería esperar un aumento elevado. En efecto, los datos muestran cierto impacto positivo en esta dimensión, si bien no es significativamente diferente.

Sin embargo, en la escala RUT (rutina de implementación) sí se observa una ganancia significativa, que podría indicar que el profesorado, tras su participación en los programas de desarrollo profesional, ha incluido en sus clases más prácticas orientadas a la indagación. Los resultados en las dimensiones relacionadas con prácticas específicas propias del IBL nos permitirá interpretar este hecho con algo más de detalle.

En efecto, los datos de las escalas relacionadas con metodologías IBL (CE-EST: centradas en los estudiantes; PRA: actividades prácticas; INV: investigaciones en el aula), muestran ganancias entre los datos del pre-test y del post-test. Sin embargo, en todas las escalas relacionadas con rutinas y prácticas los valores siguen siendo relativamente bajos (todos inferiores a 2,50), indicativos de que, más allá del impacto positivo de los programas de desarrollo profesional, queda aún un amplio margen de evolución en el 
profesorado. En concreto, es especialmente bajo el valor de la dimensión investigaciones en el aula (INV), tal vez por la complejidad que percibe el profesorado para llevarlas a cabo, y que señala un aspecto en el que el programa de desarrollo profesional debería incidir con más fuerza.

Si consideramos, de forma conjunta, la escala relativa a creencias (ORI) con las relativas a prácticas de aula (RUT, CE-EST, PRA e INV) los resultados son coherentes con los descritos en el estudio TALIS (Organisation for Economic Co-operation and Development, 2009), en el que se detectó esta paradoja entre creencias y prácticas docentes. Por un lado, el estudio identificó una alta orientación del profesorado hacia creencias de tipo "constructivista ${ }^{2}$ », más alineadas con los postulados subyacentes al IBL, frente a creencias basadas en la «transmisión directa». Sin embargo, en lo relativo a las prácticas docentes, el estudio señaló un predominio de "prácticas estructuradas» (exposición, revisión del trabajo en casa de los alumnos, control del cuaderno de ejercicios...) frente a otras que denominaban «orientadas al alumno» (trabajo en pequeños grupos, tareas graduadas en dificultad según los/as estudiantes, dar voz al alumnado a la hora de planificar el desarrollo de un tema...) o de "participación activa» (trabajo por proyectos, desarrollo de un producto, debates...), indicando, además, que este predominio era más acusado en el caso de profesorado de matemáticas y ciencias.

El considerar nuestros datos dentro del panorama más amplio que dibuja el estudio TALIS nos ayuda a comprender el reto que supone modificar y desarrollar las prácticas docentes del profesorado, incluso partiendo de un escenario favorable en relación con sus creencias. En este sentido, entendemos que las ganancias observadas en las escalas anteriores implican un trabajo en la dirección correcta y avala la pertinencia de la intervención realizada. Ahora bien, consideramos que los datos también podrían reflejar el hecho de que las intervenciones no fueron demasiado extensas (aunque la duración de los cursos fue variable, esta osciló entre 2 y 4 meses de trabajo en conjunto). En este sentido, la literatura sobre desarrollo profesional ya señala que, para que un proceso de desarrollo profesional llegue a provocar una modificación significativa en las creencias y prácticas del profesorado, es necesario que este se extienda de forma continuada en el tiempo (véase, Tirosh \& Graeber, 2003; Guskey, 2002; Loucks-Horsley et al., 2003).

Sobre las escalas relacionadas con oportunidades, barreras y dificultades que percibe el profesorado para el uso del IBL, observamos que:

- En la dimensión oportunidades que el currículo actual y el sistema educativo ofrece para el uso del IBL (LEG), el valor de partida es relativamente bajo, aunque hay un leve incremento en los valores del post-test. Este hecho es destacable, ya que entendemos que 
el marco curricular actual, así como la organización del sistema educativo, respalda de forma explícita el uso de este tipo de metodologías, aunque el profesorado perciba un escaso apoyo. Esta percepción por parte del profesorado, sin poder ser generalizada, nos indica un ámbito en el que incidir más en nuevas propuestas de desarrollo profesional.

- En la dimensión dificultades relacionadas con la gestión de una clase IBL (GEST $\left.{ }^{\text {inv }}\right)$, se detecta un valor de partida elevado en la creencia del profesorado de 2,52 $(0,43)$, lo que indica que aún un número importante de profesores lo percibe como un obstáculo para el IBL. En este caso, además, sí se aprecia un valor medio en el post-test de $2,75(0,48)$ significativamente diferente al inicial $(\mathrm{p}<0,001)$ que podría ser indicativo de que los ejemplos y estrategias metodológicas ofrecidas en el programa de desarrollo profesional para la gestión de un aula $I B L$ han tenido un impacto positivo en las barreras que el profesorado percibe sobre la gestión del aula y la recepción de esta metodología por parte de su alumnado.

- En la dimensión barreras y dificultades por la disponibilidad de recursos IBL para el aula (REC) debemos ser cautelosos en la interpretación de la significatividad ya que esta escala contenía solo dos ítems y mostraba un valor de fiabilidad moderado. Interpretamos que el programa ha sido eficaz a la hora de poner en manos del profesorado más materiales didácticos IBL, si bien queda aún un camino por recorrer ya que es una de las barreras más importantes percibidas por el profesorado.

Finalmente, en relación con las metodologías usadas en los cursos de desarrollo profesional, así como con la percepción final del profesorado implicado, y teniendo en cuenta solo la muestra post-test $(\mathrm{N}=117)$, consideramos que las ganancias descritas anteriormente en determinas creencias y prácticas docentes del profesorado que ha participado en los programas de desarrollo profesional son coherentes con su propia valoración de los mismos (ver Tabla 5, dimensiones MET-DP y REL-CDP).

En la Figura 4 se muestran los resultados relacionados con la percepción de las metodologías usadas en el programa de desarrollo profesional en cada ítem (MET-DP), expresados como la suma de frecuencias de respuestas en desacuerdo (suma de respuestas «en desacuerdo» y «totalmente en desacuerdo») y acuerdo (suma de respuestas «de acuerdo» $\mathrm{y}$ "totalmente de acuerdo»). El valor medio en la dimensión MET-DP es de 2,95 $(0,39)$. Los valores de acuerdo para los ítems de MET-DP oscilan entre el 63,4\%$94,3 \%$. Para la discusión, hemos tomado como referencia de corte $M>2,80$, que corresponde al valor de la media del ítem «diseño de investigaciones», con el fin de visualizar con más claridad qué aspectos fueron mejor o peor percibidos, ya que todos estaban por encima de la media de la escala. Así, 
los ítems mejor valorados en esta escala están relacionados con el carácter práctico del curso: oportunidades de intercambio de ideas, discusiones en grupo, actividades prácticas, discusiones centradas en la propia práctica docente, cambio percibido en la práctica docente, e intercambio de materiales didácticos. Los ítems con un valor $\mathrm{M}<2,80$ hacen referencia al intercambio y discusiones entre compañeros del mismo centro sobre materiales IBL, discusiones sobre los materiales usados en el curso, y el diseño de investigaciones y experimentos por el propio profesorado.

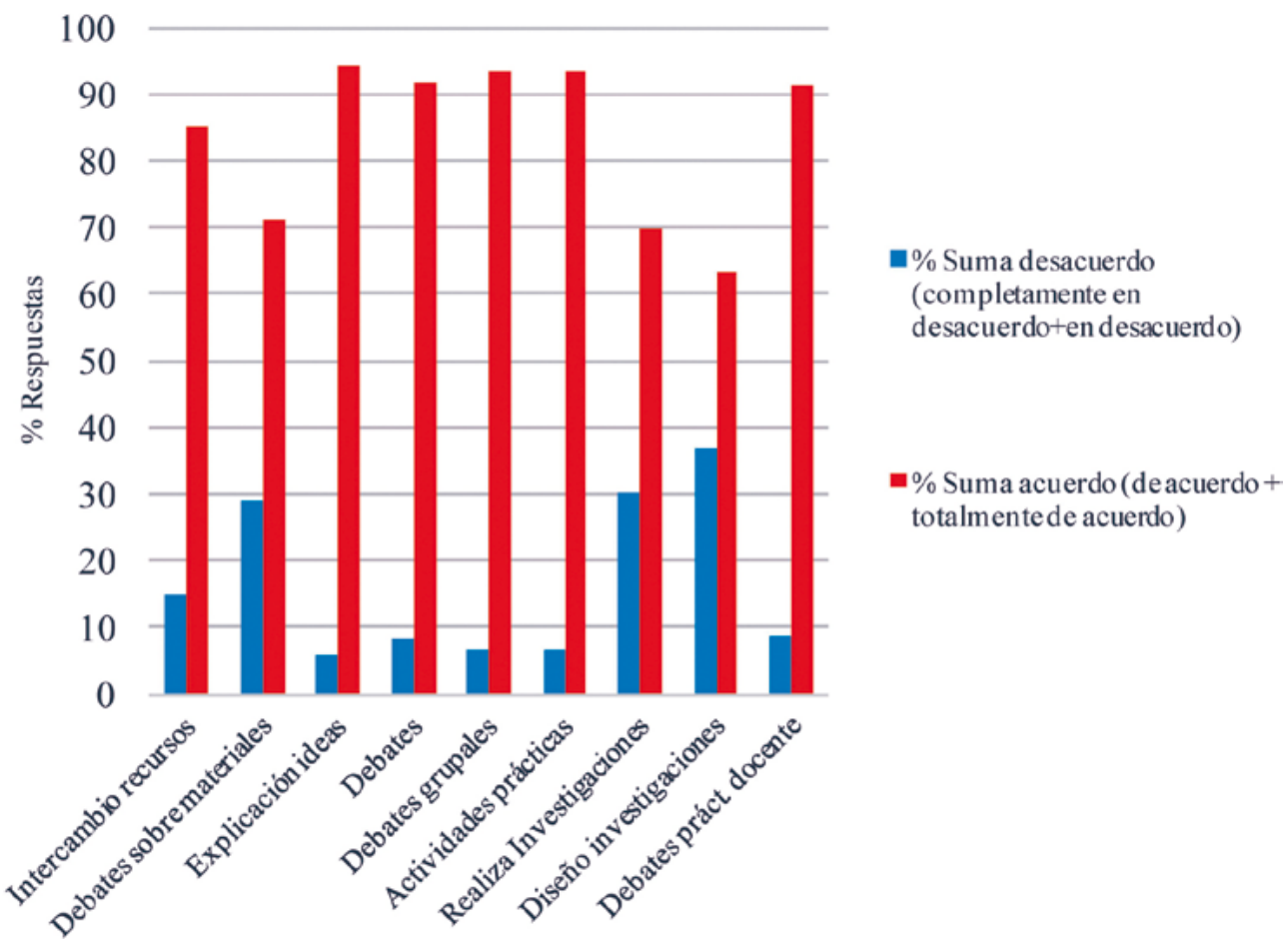

Figura 4. Percepción del profesorado de las metodologías

usadas por los formadores durante los programas de desarrollo profesional

En conjunto, la percepción de los participantes es coherente con los principios de diseño del programa de desarrollo profesional (ver apartados precedentes), que a su vez estaban extraídos de los principios identificados en la investigación respecto a programas de desarrollo profesional efectivo. Así, consideramos que nuestro estudio corrobora que un desarrollo profesional es efectivo cuando fomenta la colaboración entre el profesorado, el establecimiento de comunidades, la discusión, la realización de actividades prácticas y el diseño de investigaciones en el aula. Además, se trata de principios para el desarrollo profesional que en gran medida son reflejo de aquellos principios metodológicos que el profesorado debe 
poner en funcionamiento en clases IBL. Esta coherencia entre los procesos y contenidos del programa de desarrollo profesional ha sido también identificada en la literatura como importante para garantizar la eficacia del mismo (Tirosh \& Graeber, 2003).

La satisfacción global del profesorado respecto al programa de desarrollo profesional, medida por la escala REL-DP, fue bastante elevada, mostrando un valor medio de 3,02 $(0,40)$. Sin embargo, yendo al detalle, observamos que la media en el ítem "el curso me ha hecho cambiar mi práctica docente» es de $2,77(0,61)$, inferior a la media de otros ítems de esta escala; de hecho, un $31,1 \%$ del profesorado se muestra en desacuerdo o totalmente en desacuerdo con esta afirmación. Esta percepción arroja valores más positivos si se analiza la propia creencia sobre una mejora global en su práctica profesional (ítem «en este curso he mejorado como docente»), con un valor medio de $2,94(0,56)$.

Podemos inferir que existe un determinado sector del profesorado que, aunque no ha percibido cambios en su práctica docente, responde con una predisposición positiva a utilizar e implementar el IBL. Por ejemplo, el ítem "estoy totalmente convencido de la utilidad de este curso» arrojó un valor elevado de 3,23 (0,54), con un 95,9\% del profesorado mostrándose de acuerdo/ completamente de acuerdo. Este valor está alineado con aquel derivado del ítem "tras realizar el curso siento el compromiso de aplicar lo aprendido", $3,12(0,55)$ y $93,4 \%$ de acuerdo/completamente de acuerdo. Y aunque los cambios percibidos en rutinas y metodologías IBL sean modestos, quedando gran margen de mejora, los datos señalan que realmente el programa de desarrollo profesional ha abierto nuevas oportunidades al profesorado (ítem "he usado más situaciones $I B L$ » con un valor de $2,90(0,51)$, con un $83 \%$ de respuestas de acuerdo/completamente de acuerdo). En conjunto, todos estos valores pueden interpretarse como una predisposición positiva hacia el IBL, y son pues coherentes con los resultados obtenidos en la dimensión de orientación hacia el IBL (ORI) anteriormente descritos.

\section{CONCLUSIONES}

En este artículo hemos abordado la intersección de dos problemas de enorme complejidad. Por un lado, la necesaria revolución metodológica en la enseñanza de las ciencias y de las matemáticas hacia el IBL; por otro lado, el desarrollo profesional del profesorado, su diseño y su efectividad. Uno y otro van de la mano: no es posible una renovación metodológica sin estrategias adecuadas de desarrollo profesional, y no tiene sentido promover programas de desarrollo profesional si estos no van ligados a propuestas sólidas y renovadoras de las prácticas escolares. 
Desde el proyecto Mascil abordamos este doble reto a través del diseño y de la implementación a nivel europeo de un complejo programa de desarrollo profesional relacionado con el IBL. En este artículo hemos presentado la implementación del programa en España (18 intervenciones).

Los datos obtenidos a través de cuestionarios completados por profesorado al comienzo y a la finalización de los diferentes programas han sido reveladores en varios sentidos. Por un lado, han permitido detectar un valor alto en las actitudes y en la predisposición del profesorado hacia este tipo de metodologías IBL. Pero, por otro lado, han mostrado que sus prácticas docentes aún admiten un amplio margen de desarrollo para que estas metodologías vayan adquiriendo un peso cada vez más importante en sus prácticas de aula.

En este sentido, el programa de desarrollo profesional implementado, a pesar de los diferentes formatos usados, ha tenido un impacto positivo en esta evolución pretendida, si bien no hemos podido detectar un cambio profundo en las prácticas docentes. Teniendo en cuenta los principios de diseño adoptados, pero también la forma en la que se ha implementado en nuestro país, podemos concluir que el programa tiene más las características de un programa «transicional» que de un programa «transformativo», en el sentido de Kennedy (2014). Esta valoración queda corroborada por los datos del estudio pre-post, así como por los datos arrojados por las escalas de metodologías y relevancia del programa de desarrollo profesional (MET-DP y REL-CDP).

Finalmente, en relación con el «contenido» del programa (Tirosh \& Graeber, 2003), detectamos dimensiones especialmente importantes en las que incidir (como la relacionada con los materiales IBL a disposición del profesorado, la percepción que tienen del currículo actual, o sobre cómo llevar a cabo en el aula procesos de investigación y actividades prácticas), pero también se manifiesta cómo el profesorado va incorporando a sus prácticas docentes metodologías más centradas en los estudiantes (como el uso de cuestiones para promover el aprendizaje, o de tareas más abiertas) o cómo van superando barreras y obstáculos relacionados con la mayor complejidad de una clase IBL. En relación con los «procesos» del programa de desarrollo profesional (Tirosh \& Graeber, 2003), constatamos la importancia de fomentar la colaboración entre el profesorado, de conectar el programa con actividades prácticas y situaciones reales de aula favoreciendo los periodos de experimentación en el aula (aprender haciendo), y de compartir experiencias entre el profesorado (aprender dialogando).

Consideramos que este estudio es relevante en la medida en que ofrece claves importantes para diseñar e implementar programas de desarrollo 
profesional que pretendan ser realmente transformativos, en particular vinculados con el aprendizaje por investigación. En particular, teniendo en cuenta que una parte importante de la muestra está integrada por profesorado de primaria, los resultados sugieren la pertinencia de incorporar el aprendizaje por investigación en la formación inicial del profesorado, en concreto en el Grado de Educación Primaria. En este sentido, ya hemos realizado algunas experiencias prometedoras, como se describe en RomeroAriza, Quesada y Abril (2017).

Como limitaciones más importantes de nuestro estudio, señalamos, por un lado, la pérdida de sujetos en la muestra pareada. A pesar de no haber diferencias significativas con los resultados de la muestra no pareada, es crucial, por rigor estadístico, mejorar la recogida de datos asegurando que el mismo profesorado complete los cuestionarios pre y post. Por otro lado, la no diferenciación, en el análisis de datos y en los resultados, entre profesorado de matemáticas/ciencias y de primaria/secundaria. Aunque, de nuevo, los resultados estadísticos no muestran diferencias entre estas poblaciones, y el diseño del programa se hizo con un carácter integrador, la compresión de las diferencias entre estos colectivos puede mejorar el diseño de nuevos programas de desarrollo profesional y su implementación efectiva, acercándolos más a sus necesidades concretas y aumentando así su carácter transformativo. Finalmente, también debemos destacar el cambio modesto pero prometedor que ha experimentado el profesorado, lo que nos anima a seguir incidiendo en programas de formación vinculados con prácticas efectivas en el aula y basados en la reflexión.

\section{NOTAS}

1 Investigación financiada por la Unión Europea, proyecto $n^{\circ} 320693,7^{\circ}$ Programa Marco, 2013-2016.

2 El estudio asocia las creencias «constructivistas» con un mayor grado de acuerdo en ítems del tipo «mi papel como profesor es facilitar que mis alumnos lleven a cabo sus propias investigaciones», "los alumnos aprenden mejor buscando, por sí solos, soluciones a los problemas», o "se debe permitir a los estudiantes que busquen soluciones a los problemas prácticos por sí mismos, antes de que el profesor les muestre cómo se resuelven». 


\section{REFERENCIAS BIBLIOGRÁFICAS}

Anderson, R. (2002). Reforming Science Teaching: What Research Says About Inquiry. Journal of Science Teacher Education, 13(1), 1-12. doi: 10.1023/A:1015171124982

Artigue, M., \& Blomhøj, M. (2013). Conceptualizing inquiry-based education in mathematics. ZDM Mathematics Education, 45(6), 797810. doi: 10.1007/s11858-013-0506-6

Caprile, M., Palmén, R., Sanz, P., \& Dente, G. (2015). Encouraging STEM studies for the labour market. Brussel, Belgium: European Parliament.

Clarke, D., \& Hollingsworth, H. (2002). Elaborating a model of teacher professional growth. Teaching and Teacher Education, 18(8), 947-967. doi: 10.1016/S0742-051X(02)00053-7

Couso, D., Jiménez-Aleixandre, M.P., López-Ruiz, J., Mans C., Rodriguez Espinosa, J.M., y Sanmartí, N. (2011). Informe ENCIENDE. Enseñanza de las Ciencias en la Didáctica Escolar para Edades Tempranas en España. Barcelona: Rubes Editorial.

Donnell, L.A., \& Gettinger, M. (2015). Elementary school teachers' acceptability of school reform: Contribution of belief congruence, selfefficacy, and professional development. Teaching and Teacher Education, 51, 47-57. doi: 10.1016/j.tate.2015.06.003

Dorier, J-L., \& García, F.J. (2013). Challenges and opportunities for the implementation of inquiry-based learning in day-to-day teaching. ZDM Mathematics Education, 45(6), 837849. doi: 10.1007/s11858-013-0512-8

Engeln, K. (2017). Report on the largescale survey about inquiry-based learning and teaching in the European partner countries. Recuperado de https://bit.ly/2BNF4px

Engeln, K., Euler, M., \& Maa $\beta$, K. (2013). Inquiry-based learning in mathematics and science: a comparative baseline study of teachers' beliefs and practices across 12 European countries. ZDM Mathematics Education, 45(6), 823836. doi: 10.1007/s11858-013-0507-5

European Comission (2009). Common European principles for teacher competences and qualifications. Recuperado de https://bit.ly/2T5IFG9

Gago, J.M. (2004). Europe needs More Scientists. Brussels, Belgium: European Commission.

Glackin, M. (2016). 'Risky fun' or 'Authentic science'? How teachers' beliefs influence their practice during a professional development program on outdoor learning. International Journal of Science Education, 38(3), 409-433. doi: 10.1080/09500693.2016.1145368

Guskey, T. (2002). Professional Development and Teacher Change. Teachers and Teaching, 8(3), 381-391. doi: 10.1080/135406002100000512

Hord, S.M., \& Hirsh, S.A. (2008). Making the promise a reality. En A.M. Blankstein, P.D. Houston \& R.W. Cole (Eds.), Sustaining professional learning communities (23-40). Thousand Oaks, CA, USA: Corwin Press.

Huh, J., Delorme, D.E., \& Reid, L.N. (2006). Perceived Third-Person Effects and Consumer Attitudes on Prevetting and Banning DTC Advertising. The Journal of Consumer Affairs, 40(1), 90-116. doi: 10.1111/j.17456606.2006.00047.x

Jones, M.T., \& Eick, C.J. (2007). Implementing inquiry kit curriculum: 
Obstacles, adaptations, and practical knowledge development in two middle school science teachers. Science Education, 91(3), 492-513. doi: 10.1002/sce. 20197

Kennedy, A. (2014). Models of Continuing Professional Development: a framework for analysis. Journal of In-service Education, 40(3), 336-351. doi: 10.1080/19415257.2014.929293

Lakshmanan A., Heath B.P., Perlmutter A., \& Elder M. (2011). The impact of science content and professional learning communities on science teaching efficacy and standards-based instruction. Journal of Research in Science Teaching, 48(5), 534-551. doi: 10.1002/tea.20404

Loucks-Horsley, S., Love, N., Stiles, K., Mundry, S., \& Hewson, P. (2003). Designing professional development for teachers of science and mathematics. (2nd ed.). Thousand Oaks, CA, USA: Corwin Press.

McConney, A., Oliver, M.C., WoodsMcConney, A., Schibeci, R., \& Maor, D. (2014). Inquiry, Engagement, and Literacy in Science: A Retrospective, Cross-National Analysis Using PISA 2006. Science Education, 98(6), 963980. doi: 10.1002/sce.21135

National Research Council (2012). A framework for K-12 science education: Practices, crosscutting concepts, and core ideas. Washington, USA: National Academies Press.

Organisation for Economic Cooperation and Development (2009). Creating Effective Teaching and Learning Environments. First Results from TALIS. Recuperado de https://bit. ly/2SXwOhs

Osborne, J., \& Dillon, J. (2008). Science Education in Europe: Critical
Reflections. Recuperado de https://bit. ly/2RfBCdr

Potvin, P., \& Hasni, A. (2014). Analysis of the Decline in Interest. Towards School Science and Technology from Grades 5 Through 11. Journal of Science Education and Technology, 23(6), 784802. doi: 10.1007/s10956-014-9512-x

Rocard, M., Csermely, P., Jorde, D., Lenzen, D., Walberg-Henriksson, H., \& Hemmo, V. (2007). Science Education Now: A renewed Pedagogy for the Future of Europe. Brussel, Belgium: European Comission. Recuperado de https://bit. ly/1ixEQz5

Romero-Ariza, M. (2017). El aprendizaje por indagación: ¿existen suficientes evidencias sobres sus beneficios en la enseñanza de las ciencias? Revista Eureka sobre Enseñanza y Divulgación de las Ciencias, 14(2), 286-299. doi: 10.25267/rev_eureka_ensen_divulg_ cienc.2017.v14.i2.01

Romero-Ariza, M., Quesada, A., \& Abril, A.M. (2017). Science Teachers as Key Actors in Responsible Research and innovation: Evaluation of a teacher training program. Sisyphus. Journal of Education, 5(3), 107-121. doi: $10.25749 /$ sis. 12274

Tirosh, D., \& Graeber, A. (2003). Challenging and changing mathematics teaching classroom practices. En A.J. Bishop, M.A. Clements, C. Keitel, J. Kilpatrick y F.K.S. Leung (eds.), Second International Handbook of Mathematics Education (643-687). Dordrecht: Springer. doi: 10.1007/97894-010-0273-8_22

Thurlings, M., \& den Brok, P. (2017). Learning outcomes of teacher professional development activities: a meta-study. Educational Review, 69(5), 554-576. doi: 10.1080/00131911.2017.1281226 


\section{PERFIL ACADÉMICO Y PROFESIONAL DE LOS AUTORES}

Francisco Javier García-García. Licenciado en Matemáticas por la Universidad de Sevilla y Doctor por la Universidad de Jaén. Desde 1997 es profesor en el Departamento de Didáctica de las Ciencias de la Universidad de Jaén (área de Didáctica de las Matemáticas). Su investigación aborda, entre otras temáticas, la formación inicial y continua del profesorado. Ha participado en numerosos proyectos europeos (Erasmus y $7 .^{\circ}$ Programa Marco): Lema, Compass, Primas, Mascil y Parrise.

Antonio Quesada Armenteros. Licenciado en Ciencias Químicas por la Universidad de Jaén. Doctor Europeo. Profesor Titular de Universidad en el Departamento de Didáctica de las Ciencias de la Universidad de Jaén (área de Didáctica de las Ciencias Experimentales). Entre otras líneas, su investigación se centra en la formación inicial y continua del profesorado. Ha participado en proyectos europeos relacionados con la enseñanza de las Ciencias y las Matemáticas: Primas, Mascil, Parrise e IncluSMe.

Marta Romero Ariza. Doctora en Química Orgánica por la Universidad de Granada y Doctora Europea en 2002. Desde 2006 es profesora en el Departamento de Didáctica de las Ciencias de la Universidad de Jaén e investiga en formación de profesorado e intervenciones innovadoras para mejorar la educación en ciencias y matemáticas. Desde 2010 ha estado implicada en proyectos internacionales en esta línea (Primas, Compass, Mascil, Parrise, IncluSMe, MaSDiV y ENEC).

Ana María Abril Gallego. Doctora en Ciencias (Biológicas) por la Universidad Autónoma de Madrid. Desde 2001 es profesora en el Departamento de Didáctica de las Ciencias de la Universidad de Jaén. Sus líneas de investigación están relacionadas con la mejora de la educación científica en la formación inicial y continua del profesorado. Ha participado en proyectos internacionales tales como Compass, Primas, Mascil, Parrise, IncluSMe o MaSDiV, así como en diversos de ámbito nacional.

Dirección de los autores: Universidad de Jaén

Departamento de Didáctica de las Ciencias

Facultad de Humanidades y Ciencias de la

Educación

Paraje de Las Lagunillas s/n-Edificio D2

23071 Jaén

E-mail: fjgarcia@ujaen.es

antquesa@ujaen.es

mromero@ujaen.es

amabril@ujaen.es

Fecha Recepción del Artículo: 16. Enero. 2019

Fecha Modificación del Artículo: 18. Febrero. 2019

Fecha Aceptación del Artículo: 20. Febrero. 2019

Fecha Revisión para publicación: 25. Febrero. 2019 
